Rabaska

Revue d'ethnologie de l'Amérique française

\title{
Charlevoix est-il un pays enchanté pour Marius Barbeau ?
}

\section{Serge Gauthier}

Volume 13, 2015

Présence de Marius Barbeau : l'invention du terrain en Amérique française. Autour d'un legs centenaire (1914-2014)

URI : https://id.erudit.org/iderudit/1033745ar

DOI : https://doi.org/10.7202/1033745ar

Aller au sommaire du numéro

Éditeur(s)

Société québécoise d'ethnologie

ISSN

1703-7433 (imprimé)

1916-7350 (numérique)

Découvrir la revue

Citer cet article

Gauthier, S. (2015). Charlevoix est-il un pays enchanté pour Marius Barbeau ? Rabaska, 13, 22-29. https://doi.org/10.7202/1033745ar d'utilisation que vous pouvez consulter en ligne.

https://apropos.erudit.org/fr/usagers/politique-dutilisation/ 


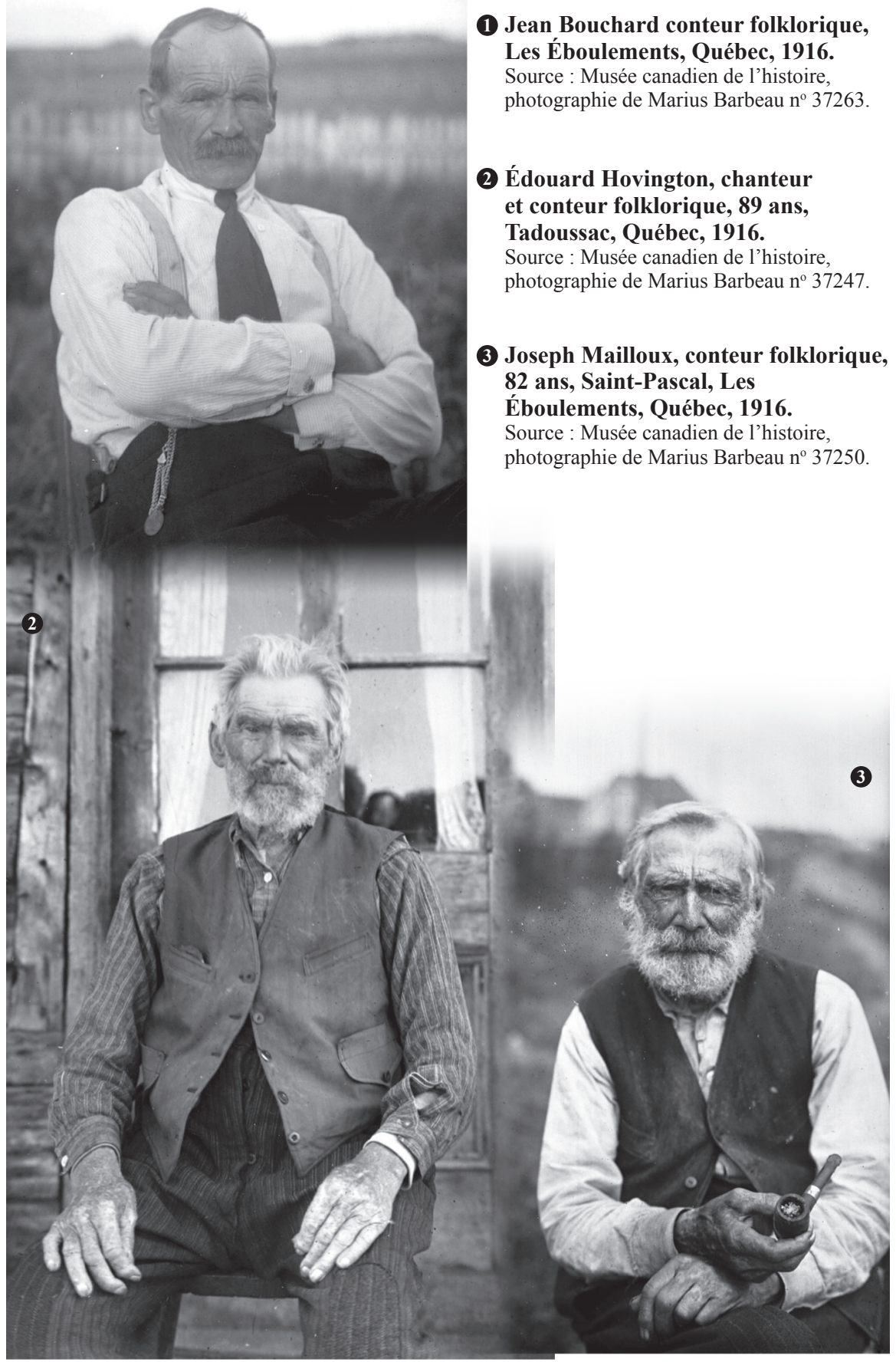




\section{Première partie}

ENQUÊTE, TERRAIN ET TERRITOIRE

\section{Le pays des gourganes}

\section{Charlevoix est-il un pays enchanté pour Marius Barbeau?}

Serge Gauthier

Société d'histoire de Charlevoix Centre de recherche sur l'histoire et le patrimoine de Charlevoix

Qu'est-ce donc que l'enchantement ? Pour Marius Barbeau, jeune folkloriste, il semble bien que ce fut cet été 1916 qu'il connut l'enchantement, alors qu'il visite ce "pays des gourganes » qu'est Charlevoix, là où cette fève des marais abonde plus que n'importe où ailleurs au Québec. Il faut reconnaître l'étendue du matériel recueilli lors de cette collecte de l'été 1916 et ainsi voir jusqu'à quel point la " découverte » de Charlevoix comme lieu d'enquête a été importante dans la carrière de Marius Barbeau. On sent aussi qu'il a pris du plaisir dans cette collecte et même parfois qu'il s'émerveille d'être dans cette région un peu magique à ses yeux et où les gens ont encore du temps pour retenir des chansons anciennes ou des contes merveilleux, même si nous sommes bien en Amérique du Nord en pleine époque moderne. Est-ce là de l'enchantement ? Sans doute.

Notre regard aujourd'hui, admettons-le, souhaite s'écarter un peu de l'enchantement. Ce sera plutôt le regard plus critique du scientifique qui revient, cent ans après, sur le parcours folklorique de Marius Barbeau dans Charlevoix. Ici la matière recueillie n'est pas notre premier intérêt, mais nous cherchons plutôt le « point de vue » où l'arrière-fond intellectuel qui a guidé Marius Barbeau dans ses collectes en Charlevoix. Car, selon que l'on est assis au fond de l'amphithéâtre ou sur le bord de la glace durant une partie de hockey, on ne voit pas le match de la même manière et, pour les chercheurs, il en va parfois de même, selon que leurs enquêtes sont liées à 
un contexte où à un autre ${ }^{1}$. Cette constatation s'impose aussi pour Marius Barbeau qui a beaucoup publié et a paru parfois se contredire ou même se dédire. Il faut voir plus loin. On a trop longtemps retenu seulement le « donné » ou la matière recueillie chez Barbeau plutôt que le contexte d'enquête'2. Plus la performance de l'informateur que la démarche de l'enquêteur. Cette approche au sujet du passage de Barbeau en Charlevoix a ainsi varié et cela influence la réception publique ou scientifique face à son travail. Parfois, il peut être considéré dépassé ou encore actuel et pertinent dans le même souffle. On ne comprend pas bien le contexte personnel et historique dans lequel il se présente. Voilà le manque que nous chercherons à combler - un peu - en lien avec son travail de recherche dans Charlevoix.

Deux textes nous guiderons pour ce trajet en Charlevoix : «Le Pays des gourganes » paru en $1917^{3}$ et The Kingdom of Saguenay ${ }^{4}$ paru en 1936 et édité en français en 1967 sous le titre Le Saguenay légendaire ${ }^{5}$.

\section{Le Pays des gourganes : le regard scientifique de Barbeau}

En fait, la conférence « Le Pays des gourganes » lue par Marius Barbeau en mai 1917 devant les membres de la Société royale du Canada n'est pas qu'un important relevé d'enquêtes folkloriques faites dans Charlevoix à l'été 1916 ; il est aussi l'un de ses premiers rapports significatifs publiés sur le folklore recueilli auprès de Québécois francophones qui marque le nouveau cheminement de Barbeau en ce domaine après ses études réalisées auprès des autochtones. Document important certes, mais aussi un peu déroutant. Surtout en ce qu'il tranche avec les parutions de Barbeau sur le même sujet, soit Charlevoix, parues par la suite. D'abord, on y note la prévalence des études sur le parler populaire local, ou relevés linguistiques, qui occupent une bonne part du texte. Par la suite, Barbeau fera moins ce type de relevé, y préférant la chanson folklorique, le conte et la culture matérielle, notamment en lien avec Jean Palardy dans Charlevoix. Mais, dans « Le Pays des gourganes », Barbeau paraît utiliser l'aspect linguistique pour planter un clou bien précis, soit que la culture traditionnelle des régions du Québec n'est pas aussi uniforme que les chercheurs du temps le disaient et qu'il y a des régionalismes. C'est une affirmation quasi révolutionnaire pour le temps, alors que les chercheurs en histoire et en folklore québécois prétendaient - bien à tort - que les « héritages culturels français »s'étaient

1. Philippe Corcuff, « Lire Bourdieu autrement », Magazine littéraire, 369, octobre 1998, p. 35.

2. Serge Gauthier, « Charlevoix ou la création d'une région folklorique. Étude du discours de folkloristes québécois (1916-1980)», Thèse de Ph. D. (Ethnologie historique), Université Laval, 2004. $235 \mathrm{p}$.

3. Marius Barbeau. «Le Pays des gourganes », Mémoires de la Société Royale du Canada, série 3, vol. 11, no 1, 1917, p. 193-225.

4. Marius Barbeau, The Kingdom of Saguenay, Toronto, MacMillan, 1936, [12]-167 p.

5. Marius Barbeau, Le Saguenay légendaire, Montréal, Beauchemin, 1967, 147 p. 
maintenus sans changements ou presque depuis la Conquête (1759) chez le peuple québécois. En fait, il peut paraître étonnant que ce soit Marius Barbeau qui tente de défaire cette vérité discutable, et pourtant c'est bien lui.

Marius Barbeau n'y va d'ailleurs pas de main morte en ce qui concerne le « pays des gourganes »: " Le Pays des gourganes [Charlevoix] n'ayant jamais été isolé que relativement, il a de tout temps été en contact avec les régions avoisinantes ${ }^{6} »$. Voilà qui a l'avantage d'être clair. En 1934, dans son livre Au cœur de Québec, Barbeau semble pourtant affirmer tout le contraire : "L'isolement de ce district montagneux et fluvial [Charlevoix] lui a servi de sommeil enchanté, comme dans le conte. Il a dormi deux cents ans. ${ }^{7} \gg$. En moins de vingt ans, Barbeau paraît donc se contredire grandement. Nous postulons toutefois ici que ce changement est dû au positionnement du regard de Barbeau, selon les époques de sa vie, qui accordent à ses relevés d'enquête dans Charlevoix un cadre intellectuel différent, voire divergent. Il faut parler dans le cas de l'article « Le Pays des gourganes » d'une approche scientifique de ses relevés de recherche dans Charlevoix.

Destiné à un public de chercheurs, "Le Pays des gourganes » cherche essentiellement à affirmer l'existence de régionalismes au Québec. Jeune chercheur, Barbeau y voit l'occasion de se démarquer. Le coup va porter. L'intérêt des autres chercheurs sera grand et personne ne pourra voir cette démarche de collecte de la même manière après Barbeau qui a créé littéralement ici l'enquête folklorique de terrain de type scientifique au Québec.

Pour Charlevoix ou le «pays des gourganes », le regard scientifique de Barbeau dans son article de 1917 donnera le point de vue suivant :

\section{Tableau 1}

\section{Regard scientifique de Barbeau dans « Le Pays des gourganes » en 1917}

1) Charlevoix n'est pas une région isolée et a donc des liens avec les régions voisines qui influencent sa culture régionale.

2) Charlevoix comme région est placée dans l'histoire et des faits comme l'émigration des Québécois vers les États-Unis ou la migration des Charlevoisiens vers le Saguenay possèdent leur importance sur la variation des données folkloriques recueillies.

3) Le matériel folklorique recueilli sur le terrain du " pays des gourganes » n'est nullement spécifique, ni totalement reçu intact de France depuis la Conquête anglaise, ni totalement distinct de celui des régions avoisinantes, et donc il s'agit pour ce territoire d'un isolat « relatif» ou n'existant sans doute même pas.

6. Marius Barbeau, «Le Pays des gourganes », op. cit., p. 195.

7. Marius Barbeau, Au cœur de Québec, Montréal, Édition du Zodiaque, 1934, p. 34. 
Certains pourraient s'étonner - même aujourd'hui - de ces affirmations de Barbeau et pourtant elles sont contenues dans l'article « Le Pays des gourganes ». Est-ce qu'il les a rejetées par la suite ? Nous pensons que non et qu'elles sont demeurées importantes pour lui. Elles restent la conviction initiale du Barbeau au regard scientifique et il ne les renie jamais. Nous pensons plutôt qu'il les a placées - en ce qui concerne Charlevoix - dans un autre contexte et qu'à cause de la diffusion importante de ces données en milieu touristique, la visée initiale en a été quelque peu éludée et pourtant, nous le pensons, elle n'a jamais disparu.

\section{The Kingdom of Saguenay : le regard touristique de Barbeau}

Comme cela était son droit, Marius Barbeau a poursuivi diverses associations et entreprises au cours de sa longue carrière. L'une des plus fructueuses d'entre elles fut son association avec William Coverdale, le président de la Canada Steamship Lines, une entreprise maritime qui offre au grand public la célèbre « croisière du Saguenay ». Il en ressort notamment le livre The Kingdom of Saguenay publié par Barbeau en 1936 qui fut un véritable guide de voyage pour tous les croisiéristes du «Tour du Saguenay » ou du «Saguenay Trip ». Le livre fut vendu régulièrement dans le cadre de la croisière et souvent réédité jusqu'à la fin de cette activité touristique en 1965. Un grand succès qui impose aussi le regard estivant et anglophone qui vient avec, puisque ce groupe constitue la grande majorité des clients de la croisière. Une édition banale et assez mal traduite de The Kingdom of Saguenay est parue en français longtemps après, en 1967, et il est possible de se demander si Barbeau alors très âgé (il allait mourir en 1969) l'a vraiment révisée. En fait, la qualité n'est plus vraiment là. Notons que The Kingdom of Saguenay - comme d'ailleurs la version française, qui l'est aussi, mais avec moins d'illustrations et moins bien présentées - est illustré par des scènes pittoresques en provenance de peintres amis de Barbeau, notamment des membres du Groupe des Sept. Il faut noter aussi que le Royaume du Saguenay de Barbeau exposé dans cet ouvrage concerne essentiellement Charlevoix, car le folkloriste n'a pas fait de recherches de terrain très approfondies dans le secteur du Saguenay, mais essentiellement dans Charlevoix.

Mais que contient The Kingdom of Saguenay? En fait, c'est encore le produit des enquêtes de Barbeau de 1916 dans Charlevoix, mais plus encore, puisque nous sommes en 1936 et que Barbeau a fait d'autres collectes dans Charlevoix depuis. Le registre est ici plus littéraire que scientifique et l'on sent que Barbeau s'amuse à écrire plus qu'il ne souhaite refléter une démarche scientifique quelconque. Il y retient particulièrement un ton légendaire glissant vers le conte la plupart du temps ; il n'y a pas de descriptions 
de chansons folkloriques dans The Kingdom of Saguenay. Les sujets retenus de ses enquêtes de terrain deviennent ainsi de petits contes amusants, notamment sur Louis l'Aveugle, le père de La Brosse et le fameux Alexis le Trotteur dont Barbeau est le principal découvreur littéraire en quelque sorte. Certains récits sont issus de l'histoire, mais traités de manière irréelle, comme le passage de Jacques Cartier au Saguenay en 1535 et la division de la Seigneurie de La Malbaie entre deux seigneurs écossais après la Conquête. Il ne faut surtout pas prendre ces textes comme des documents historiques, ce qui fut fait trop souvent malheureusement par de nombreux chroniqueurs et même des historiens. Un chapitre de Kingdom of Saguenay est particulièrement étrange, soit celui du « Père Raquette » où Barbeau transforme le peintre A.Y. Jackson, son collaborateur et ami, en personnage légendaire en quelque sorte. Plutôt audacieux nous en conviendrons, mais pas très ethnologique, et d'ailleurs, très curieusement, ce texte destiné à un public anglophone ne fut pas traduit en français dans Le Saguenay Légendaire et on se demande un peu pourquoi. Seul Barbeau et ses éditeurs pourraient le dire.

Si l'on veut résumer le point de vue lié à la démarche à optique touristique de Barbeau on retiendrait le tableau 2 qui suit :

\section{Tableau 2}

\section{Regard touristique de Barbeau dans The Kingdom of Saguenay}

1) Charlevoix (Royaume du Saguenay) devient une région isolée et fermée sur elle-même, ce qui assure son intérêt folklorique et se marie avec l'optique de la croisière qui cherche à présenter la région comme spécifique et isolée.

2) L'approche est « hors du temps ». Barbeau parle souvent de l'époque médiévale comme comparatif au vécu de Charlevoix en ce début de $\mathrm{xx}^{\mathrm{e}}$ siècle, ce qui est une volonté très claire de ne pas envisager la réalité afin de plaire au regard des touristes. Le ton est souvent colonial et l'on sent presque que les habitants du « Royaume du Saguenay » ne sont pas des êtres historiques.

3) Les références folkloriques semblent associées à ce secteur de manière totalement originale afin de favoriser l'identification du touriste à la région « sauvage » et isolée que les responsables de la croisière du Saguenay prétendent lui faire visiter. La discussion sur les liens entre régions voisines et le régionalisme réel est ici totalement disparue.

On conviendra que nous ne sommes pas ici dans le meilleur du Marius Barbeau, ce chercheur scientifique universitaire reconnu internationalement. Faut-il le lui reprocher? Non. Il avait le droit de publier des ouvrages plus légers, moins sérieux. Ce qui est dommage cependant, c'est que la grande 
diffusion de ses documents à visée touristique a fini par faire prédominer cette approche sur celle du Barbeau scientifique de l'article « Le Pays des gourganes » en ce qui concerne Charlevoix. Nous pensons que l'approche du Barbeau scientifique aurait dû se maintenir, mais elle n'a pas bien résisté, même si elle était juste scientifiquement, devant les affres du tourisme de masse de la populaire « croisière du Saguenay » organisé et prédominant durant de nombreuses années.

\section{Prendre le relais de Marius Barbeau}

Il faut pourtant prendre le relais de Marius Barbeau. Le relevé de ses collectes déposées au Musée canadien de l'histoire, en ce qui concerne Charlevoix et d'autres régions québécoises francophones, est unique et exemplaire. Ses livres, bien sûr, même nombreux, n'en rendent qu'une infime partie, mais cela ne justifie pas que l'on oublie cet héritage unique.

Plusieurs chemins peuvent conduire à la mise en valeur de l'œuvre de collecte de folklore de Barbeau, et spécifiquement celle recueillie dans Charlevoix. Certains s'y sont tenus rigoureusement comme ses successeurs immédiats des Archives de folklore : Luc Lacourcière, Félix-Antoine Savard, Conrad Laforte dans le domaine de la littérature orale et Jean-Claude Dupont en culture matérielle. Mais d'autres, plus récemment, prennent davantage la voie touristique aussi empruntée par Barbeau et ne conservent qu'une surface un peu artificielle et sans vraiment de mise en contexte historique de son héritage : Alexis le Trotteur et son squelette devenu touristique, maintenant retourné en terre heureusement ; musées régionaux et économusées comme propositions touristiques ; programme d'ethnologie où l'étude du tourisme l'emporte sur celle plus traditionnellement reconnue de la littérature orale; livres d'art à grand tirage bellement imprimés, mais souvent peu rigoureux sur le plan historique. À quand des études sur la démarche ethnologique auprès des francophones du Québec dont le travail serait plus théorique que visuellement attrayant ? À ce jour, nous restons peut-être un des seuls à le faire avec notre doctorat intitulé Charlevoix et la création d'une région folklorique ${ }^{8}$. Il y a là des relais à prendre, nous le pensons vraiment.

Dans Charlevoix, nous tenons le flambeau avec le Centre de recherche sur l'histoire et le patrimoine de Charlevoix : plusieurs de nos recherches publiées dans la Revue d'histoire de Charlevoix ont utilisé l'enquête orale de terrain comme Barbeau l'a fait autrefois. En tenant toutefois compte du contexte historique, comme Barbeau dans « Le Pays des gourganes » et sans pour cela dénier l'importance touristique de Charlevoix. Mais, en donnant à

8. Serge Gauthier, Charlevoix ou la création d'une région folklorique. Étude du discours de folkloristes québécois (1916-1980), Québec, Presses de l’Université Laval, 2006, XIII-208 p. 
cette " culture de l'Autre » une deuxième place, pas une première dans une région où le peuplement sédentaire français commence il y plus de 350 ans et non avec 200 ans de villégiature surtout anglophone. Sommes-nous des successeurs de Barbeau? Qui sait?

En terminant, nous sommes convaincu d'une chose : les données folkloriques recueillies par Marius Barbeau il y a déjà plus de cent ans ont encore un sens aujourd'hui. Pour les rendre actuelles et intéressantes pour les chercheurs de demain, il faut leur octroyer un temps de réflexion et d'analyse, orienté davantage sur leurs présupposés intellectuels ou le point de vue qui les régissait que sur leur contenu. Cette matière unique témoignant d'un passé, peut-être même révolu à l'époque de Barbeau, rayonnera ainsi de nouveau et d'une autre manière. Les générations à venir sauront assurément s'en souvenir et remercier ce chercheur étonnant que demeure Marius Barbeau pour avoir sauvé de la disparition tant d'héritages folkloriques recueillis dans Charlevoix ou ailleurs en Amérique française, pour n'en rien oublier comme cela est nécessaire et plus urgent que jamais.

Finalement, Charlevoix était-il un pays enchanté pour Marius Barbeau? Il semble bien que ce soit le cas. Nous espérons ainsi que ce terrain de collecte si important pour Marius Barbeau le demeure encore aujourd'hui pour d'autres chercheurs et pour ceux qui viendront après eux. 